\title{
High-Resolution 3D Reconstruction of Cytoplasmic Polyhedrosis Virus
}

\author{
Yuyao Liang ${ }^{*}$, Joanita Jakana ${ }^{* *}$, Xue-Kui Yu*, Jing-Qiang Zhang ${ }^{* * *}$, Wah Chiu ${ }^{* *}$, Z. Hong Zhou \\ *Department of Pathology and Laboratory Medicine, University of Texas - Houston Medical School, \\ Houston, TX 77030, USA \\ ** Department of Biochemistry, Baylor College of Medicine, Houston, TX 77030, USA \\ ${ }^{* * *}$ State Key Lab for Biocontrol, Zhongshan University, Guangzhou, 510275, China
}

\section{Introduction}

A $300 \mathrm{kV}$ electron cryomicroscope equipped with a liquid helium-cooled specimen stage and a field emission gun (FEG) electron source was used to image a dsRNA virus. The improved coherence of the electron beam and the increased electron dosage allowable for imaging contributed together to the signal/noise ratio enhancement at high resolution. Such improvements have made it possible to image ice-embedded macromolecular complexes to $4.5 \AA$ resolution (Fig. 1a-c). In this pilot study, cytoplasmic polyhedrosis virus (CPV) was chosen for the high-resolution threedimensional (3D) reconstruction. CPV is an insect pathogen and belongs to the Reoviridae family, which is a group of segmented, dsRNA icosahedral viruses that infect mammals, insects and plants. Differing from most reoviruses that are either double-shelled capsids or triple-shelled capsids, infectious CPV is a single-shelled capsid and is thus, the structurally simplest member among these viruses. Furthermore, CPV is one of the most stable viruses and can be easily purified by density gradient. Thus, CPV provides an ideal model for high resolution imaging and 3D reconstruction, as well as for the study of structural basis of dsRNA virus replication [1].

\section{The CPV Structure}

CPV particles were purified from polyhedra as described in previous paper [1]. Focal pairs of micrographs with 60k magnification were recorded for each specimen area with the first micrograph at a close-to-focus (e.g., $0.2-1.7 \mu \mathrm{m}$ ) condition, followed by a far-from-focus micrograph (e.g., 1.9-3.7 $\mu \mathrm{m}$ ). Power spectrum analyses of these micrographs, which were digitized with step size of $1.17 \AA /$ pixel on the specimen scale, demonstrate that image resolutions better than $4.5 \AA$ were obtained (Fig. 1b-c). To process these high resolution images, we have developed an integrated software package with an image database, the Image Management and Icosahedral Reconstruction System (IMIRS), in the highly available, high performance Windows XP environment [2]. A 3D reconstruction of the full CPV was obtained by merging 14953 close-tofocus particles from 237 micrographs at $5.5 \AA$ resolution (Fig. 1d). The structure reveals the structural organization of the CPV capsid with unprecedented details and has allowed the identification of a large number of $\alpha$ helices and $\beta$ sheets in the three proteins that make up the capsid shell. The majority of these secondary structures in turret protein (TP) are topologically equivalent to those observed in the x-ray structures of the corresponding proteins in other multilayered reoviruses. However, major differences were evident at the outermost region of TP, including a completely lack of the three small immunoglobulin like domains.

\section{References:}

○ [1]. Zhang H et al., Virology, 298 (2002), 45-52.

- [2]. Yuyao Liang et al., Journal of Structural Biology, 137(2002) 292-304.

$\circ$ [3]. This research is supported in part by US Public Health Services (AI46420, CA94809 to 
ZHZ; Grant \# PR 02250 to WC) and the Robert Welch Foundation (AU-1492 to ZHZ). ZHZ is a Pew Scholar of Biomedical Sciences and a recipient of an Established Investigator award from the American Heart Association (\#0240216N).
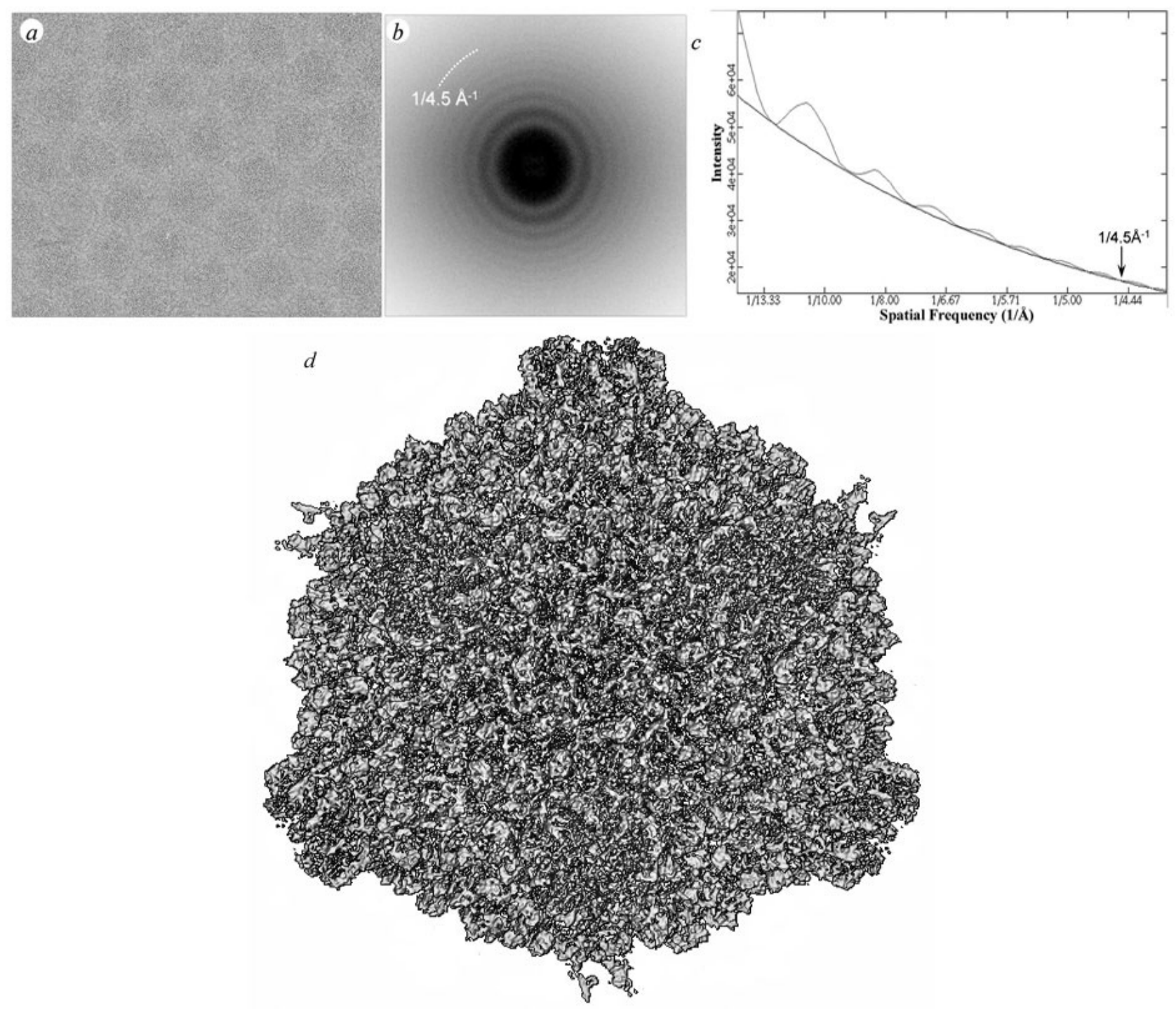

Figure 1. High resolution imaging and 3D reconstruction of the CPV. (a) Close-to-focus micrograph. (b) Incoherently averaged Fourier transform of the close-to-focus. (c) CTF curve of the close-to-focus micrograph. (d) Shaded surface of CPV representation as viewed along an icosahedral threefold axis. 